\title{
Is Endometrial Ablation still a valid option in the management of abnormal uterine bleeding?
}

\author{
Z Abdool \\ Department of Obstetrics and Gynaecology, University of Pretoria, Pretoria, South Africa
}

\begin{abstract}
History of the study of uterine cavity
Although the interior of the uterus has been studied for more than two centuries by pioneers using mirrors, specula and candles, modern hysteroscopy was practiced from the early 1900's when David described the technique, the indications and contraindications. ${ }^{1}$ Hysteroscopy is now considered the gold standard not only for visualizing the cervical canal and uterine cavity but also for treating different benign pathological endometrial conditions. In the 1980s-1990s there were only two options for performing endometrial biopsies - blind procedures (dilatation and curretage or use of endometrial suction biopsy curettes) or targeted hysteroscopic biopsies (THB). The former could not invariably confirm a definitive diagnosis and the latter still required dilatation of the cervical canal and some form of anaesthesia. Most diagnostic hysteroscopes had a lens diameter of $5 \mathrm{~mm}$ or more but the most recent hystereoscopes are based on a $2 \mathrm{~mm}$ rod lens system within a $4 \mathrm{~mm}$ sheath. Hysteroscopy and endometrial biopsy is now the first-line investigation of women with abnormal uterine bleeding since it allows direct visualization of the endometrial cavity and the opportunity to obtain a directed endometrial biopsy.
\end{abstract}

\section{Abnormal uterine bleeding}

Understanding the physiology of the normal menstrual cycle is imperative when evaluating a women with abnormal uterine bleeding (AUB). The normal menstrual cycle is characterized by its length (mean $28 \pm 7$ days), duration of flow (mean $4 \pm 2$ days) and amount of blood loss (mean $40 \pm 20 \mathrm{ml}$ ). AUB is characterized by changes in either the frequency or duration of flow or the amount of blood loss. AUB is usually defined as dysfunctional uterine bleeding (DUB) when pelvic pathology or underlying medical causes have been excluded, it commonly manifests itself in the absence of ovulation. ${ }^{2}$

Menorrhagia is heavy cyclical menstrual bleeding, whereas menometrohagia is heavy irregular menstrual blood loss over

\section{Correspondence:}

Dr Zheela Abdool

Email: evanlogg@medic.up.ac.za several consecutive cycles. When the monthly blood loss exceeds $80 \mathrm{ml}$ it is considered excessive since approximately two thirds of women develop iron deficiency anaemia. ${ }^{3}$ Menorrhagia is a significant health care problem both for women in the developed world with one in 20 women aged 30-49 consulting their general practitioner with this complaint each year in the United Kingdom $(\mathrm{UK})^{4}$ and the developing world where consequences of iron deficiency anaemia are more prominent. Decreased quality of life (social activities hobbies, holidays) soon follows. Approximately 20\% of women in the UK will undergo a hysterectomy by the age of 55, mostly for menorrhagia . As many as two million women (22\%) each year will consult a physician for menorrhagia in the United States and approximately seven hundred thousand hysterectomies are performed each year in the United States for symptomatic menorrhagia. $^{5}$

Menorrhagia can be defined either objectively, when the blood loss is quantified or subjectively, based on the women's complaint of excessive menstrual blood loss. Quantification of menstrual blood is very inaccurate and not performed in clinical practice. Pictorial charts are also seldom used. Counting the number of standard pads of tampons used during an average menstrual cycle may offer an indication of blood loss. When more than thirty pads or tampons are soaked per menstrual period further investigation is warranted. In practice the diagnosis is mainly based on the women's subjective assessment of blood loss. The conventional first line of treatment is medical with nonsteroidal anti-inflammatories, progestins, danazol, gonadotropin releasing hormone agonists, antifibrinolytics or insertion of a progestogen releasing intrauterine device forming part of an array of therapeutic options. Endometrial ablation has been developed in the early 1980s as an alternate therapy in these women, less radical than a hysterectomy and possibly more effective than medical treatment (Table I)

Hysterectomy leads to cessation of menstruation but 35-50\% of uterine specimens show no histological abnormality. ${ }^{6}$ This suggests that the majority of hysterectomies performed for menorrhagia may be unnecessary. ${ }^{7}$ Hysterectomy is the second most common major operation performed in the United States today. It is associated with operative morbidity, cost and protracted postoperative convalescence. With this background there has been interest in the development of less invasive and less expensive treatment for heavy 


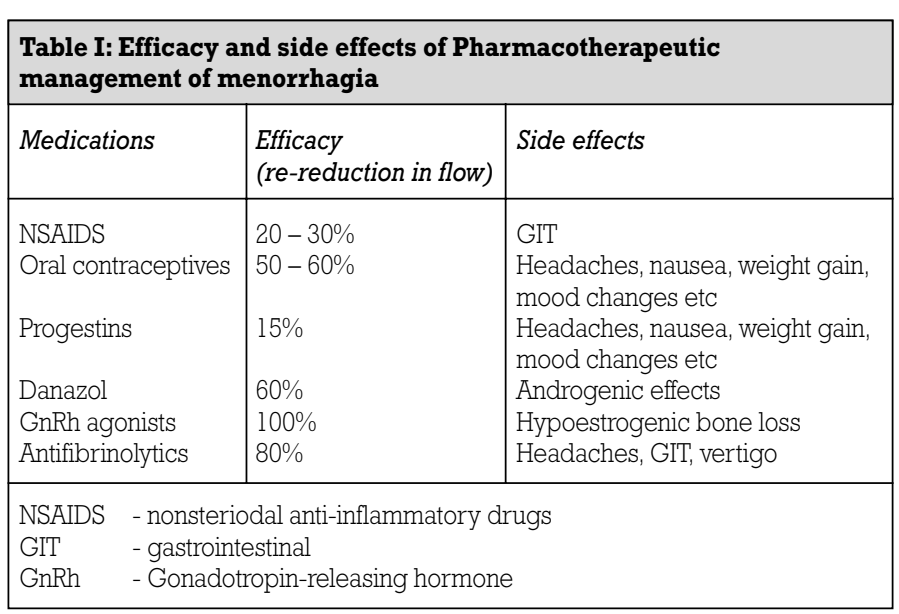

menstrual blood loss. A less invasive procedure in which the endometrial lining is destroyed but the uterus preserved would be beneficial to patients with menorrhagia.

\section{Medical and hormonal treatment}

Hormone therapy will benefit approximately $65 \%$ of patients. High dose estrogen can be used initially to treat women who are haemodynamically stable. The result is rapid regrowth of the endometrium and an increase in fibrinogen and Factors 5 and 9, with promotion of clotting and platelet aggregation at the capillary level. The risk of deep vein thrombosis with high dose estrogen exists. A low dose contraceptive pill can be prescribed after the bleeding has been controlled. The quantity of menstrual flow is decreased by $50-60 \%$ when oral contraceptives are used longterm.

NSAIDS inhibit endogenous prostaglandin production. Women with excessive menstrual blood loss exhibit higher levels of prostaglandins. The use of NSAIDS should begin on the first day of the menstrual cycle and continue to the third day of menses. Dosages vary with the type of medication. Antiprostaglandins are effective in 20-30\% of women experiencing menorrhagia.

GnRh agonists induce a medical menopause. They are effective for short term use especially in the intranasal form. Long-term use is associated with hypoestrogenic side-effects and bone loss

Androgenic steroids like danazol are expensive, have significant side effects and are rarely used for this indication.

Medical treatment is only effective in approximately 50\% of women seeking treatment. The Royal College of Obstetricians and Gynaecologists (RCOG) guidelines recommend that drug treatment should be given for at least three cycles before considering another treatment option.

The levonorgestrel-releasing intrauterine system(LNG-IUS) is also effective in the treatment of dysfunctional uterine bleeding with a reported reduction of menstrual blood loss of $97 \% .{ }^{8}$ It induces endometrial atrophy and has a Pearl index of 0,11. Over a twelve month follow up it has been shown to be as beneficial as hysterectomy for health related quality of life at less than a third of cost. ${ }^{9}$ In a randomized study of fifty women with menorrhagia the LNG-IUS was found to be as effective as endometrial thermal balloon ablation when measured on a pictorial menstrual chart. ${ }^{10} \mathrm{~A}$ meta-analysis of trials comparing the LNG-IUS with first generation endometrial ablative techniques show that the LNG-IUS is associated with a smaller reduction in blood loss and a lower amenorrhoea rate than ablation although similar satisfaction rates have been reported. ${ }^{11}$

\section{The success of treatment}

The success of treatment depends largely on whether patient expectations have been met. Patients expecting amenorrhea should consider a hysterectomy as this is the only procedure that can guarantee such a result immediately and permanently. If the expectation is to experience normal or near normal menstrual blood loss less radical treatment can be offered. This would include endometrial ablation. The choice of surgical treatment should be made jointly by the women and clinician after an informed discussion on the desired outcome, relative benefits of all other treatment options and adverse events associated with them, as well as the clinical condition, anatomical suitability and preferences of the women.

\section{Endometrial ablation}

When surgery is considered for management endometrial ablation (EA) can be considered in the first place. Endometrial ablation is the elimination of the endometrium by thermal energy or resection. The aim is to destroy the full thickness of the endometrium and also the endometrial glands in the superficial myometrium. EA preserves the uterus and is less costly and less invasive than hysterectomy. It is designed to treat AUB in women with no intrauterine pathology. Interest in ablation procedures stemmed from studying patients with Asherman syndrome. Since the description of hysteroscopy by Lindemann in 1971, several methods of endometrial ablation using hysteroscopic guidance have been developed.

Complete destruction is one of the most important determinants of treatment success. EA should not be offered as a therapeutic option for those requesting amenorrhoea but rather for well motivated women requiring management of incapacitating menorrhagia whose primary consideration is return to normal flow or less.

In recent years, endometrial ablation has become a wellestablished treatment as an alternative to hysterectomy. Despite the technique becoming an established and accepted part of practice, audits of surgical practice in the United States and the United Kingdom have not demonstrated an expected decrease in hysterectomy rates for dysfunctional uterine bleeding. It has been estimated that less than 20000 endometrial ablations are done annually in the USA compared with 700000 hysterectomies. ${ }^{12}$ This ratio of more than 1 to 30 in favour of hysterectomy may suggest that women are not being given the opportunity to consider a less invasive technique or a reluctance to prefer surgical advances above existing procedures.

\section{Endometrial ablation techniques}

The first generation techniques introduced almost twenty years ago include endometrial resection, rollerball electrocoagulation and laser ablation. The most widely used techniques are transcervical resection of the endometrium (TCRE) using a loop diathermy electrode and rollerball (RB) ablation using an electrode with a movable ball. All the first generation endometrial ablation techniques (FEATs) require direct endometrial visualization with a hysteroscope. The success rate is largely dependent on operator skill and experience. Five year amenorrhoea rates of 50\& $1 \%$ can be expected but menstruation may resume after some time.

Laser coagulation (ND-YAG) also requires distension of the uterus. The endometrium is coagulated under hysteroscopic control. It is currently least practiced due to high cost, complications and levels of hysteroscopic skills required.

TCRE- This technique was first described by DeCherney in 
1987.In TCRE the endometrium is systematically resected from the uterine fundus with attention to the cornual areas followed by a rollerball device to cauterize the bleeders.

RB: The RB was first described by Lin et al in 1988. It evolved to be commonly used because it is easier to use and is associated with less risks and complications than the loop electrode. In the MISTLETOE study RB ablations were associated with the least operative and postoperative complications. ${ }^{13}$

Adverse events include electrosurgical burns, uterine perforation, haemorrhage, infection, fluid overload (which may cause congestive cardiac failure, hypertension, haemolysis, coma and death). Mortality from the first generation ablation techniques was shown to be 0.26 per 1000 procedures. ${ }^{13}$

Although the FEATs have been described as the most thoroughly evaluated new surgical treatment option, few gynaecologists are skilled in the technique and the number of FEAT procedures has decreased in most countries. This led to the development of the second-generation EA techniques (SEATs) with the aim of providing simple, quicker and more effective treatment options for heavy menstrual bleeding compared with FEATs and hysterectomy.

SEATs are less operator dependent than the FEATs but rely largely on instrumentation to ensure safety and efficacy. It is also referred to as global endometrial ablation and is defined as automated destruction of the endometrium with an energy source without the use of operative hysteroscopy. There are two exceptions to this: Hydrothermablation can be performed under hysteroscopic observation and the microwave endometrial ablation is operator dependent and not automated.

SEATs include fluid-filled thermal balloon EA (TBEA), radiofrequency (thermoregulated) balloon EA, microwave EA (MEA), cryoablation and laser. The most frequently used technique in UK clinical practices are the fluid-filled TBEA and MEA. These techniques do not require visual inspection of the endometrium and can be done under local or general anaesthesia. Most SEATs will result in about 30-50\% of women having amenorrhoea at 6 months postoperatively and approximately 80-90\% reporting satisfaction.

The hot-liquid balloons include the Thermachoice ${ }^{1,2,3}$, the CavaTerm, CavaTerm Plus and Thermablate. In the fluid-filled TBEA technique heated liquid within a silicon balloon is inserted into the uterine cavity, and inflation ensures contact between the heated fluid and endometrium. Temperatures of $75-85^{\circ} \mathrm{C}$ are used for $5-15$ minutes. The Thermachoice has a heating element and thermistor within the balloon whereas the CavaTerm system heats saline or glycine within a separate unit and circulate it within the balloon. CavaTerm is contraindicated in women whose uterus is more than $10 \mathrm{~cm}$ long (from internal os to the fundus) and ThermaChoice for women whose uterine cavity is more than $12 \mathrm{~cm}$. TBEA should not be performed in a woman with a previous classical caesarian section or where previous uterine surgery has left a myometral thickness of less than $8 \mathrm{~mm}$ thick.

The ThermaChoice system has been widely used and tested. The pilot study on 30 patients was performed in 1994. In 2004 the instrument has evolved from ThermaChoice 1 to 3 - it was modified to allow better expansion of the balloon and coverage of the uterine cavity. The first published randomized trial (Meyer 1998) ${ }^{14}$ in which the ThermaChoice was compared to the $\mathrm{RB}$ ablation was a landmark study to validate the new second generation techniques. There was a decrease in the use of general anaesthesia and lower average procedural time of the balloon therapy versus the rollerball technique.
The first long term follow-up report of a SEAT (ThermaChoice) was published in 2003. ${ }^{15}$ Follow-up data were presented at 4-6 years from a cohort of women who were previously treated with TBEA for menorrhagia. Of the 260 questionnaires sent 188 replied. The primary outcome was avoidance of hysterectomy. The results were: the probability of avoiding hysterectomy was $86 \%$ for all women and avoiding re-abalation was $88 \%$ of non-hysterectomised women. Overall the probability of avoiding surgery was 75\%. The high rate of avoiding hysterectomy at 4-6years was encouraging. The ThermaChoice system has been evaluated as an outpatient procedure in an observational study consisting of 53 women. ${ }^{16}$ The procedure was successfully completed in $94 \%$ of patients. One failure was the result of equipment breakdown, another was a technical failure due to cervical stenosis and the other was abandoned due to severe discomfort. The procedure was well tolerated by most women. Although 14\% experienced severe discomfort only 2 of 7 would have preferred general anaesthesia with hindsight. All patients received diclofenac, 100mg rectal suppository; plus two co-dydramol tablets orally and odansetron $4 \mathrm{mg}$ orally.

The use of microwaves to treat menorrhagia was introduced in 1995 by Sharp et al. With MEA, a frequency of $9,2 \mathrm{GHz}$ penetrates to a depth of $6 \mathrm{~mm}$ from a hemispherical tip of an intrauterine probe. The displayed temperature remains between $75-80^{\circ} \mathrm{C}$ as the probe is moved slowly side to side for 3-6 minutes depending on cavity size. Advantages include short time (3-4min) and applicability up to $14 \mathrm{~cm}$ cavity length. Disadvantages include requirement for pretreatment of the endometrium, cervical dilatation up to $9 \mathrm{~mm}$ and an ultrasonic measurement of myometrial thickness. It is the only SEAT that is operator dependent. The overall complication rate is 1,5\%.

Other SEATs include devices with radiofrequency thermal energy applied globally within the uterine cavity,cryosurgery which involves freezing the endometrium for two freeze-thaw cycles of 5-7 minutes and photosensitizing drugs to induce cellular necrosis (clinical studies only, no commercially available system).

The features of an ideal ablative technique is described in Table II

\section{Table II: Features of ideal ablation technique}

Operator skill

Easy to learn with short learning curve

No operative hysteroscopic skills needed

Possible to learn technique on simulator

Effectiveness and versatility

High rate of amenorrhoea independent of operator skill and experience

Suitable for women with large uterus or uterine cavity distorted by fibroids

\section{Safety}

Very low rate of complications

Impossible to activate device if uterus is perforated

Outcome and complication data easy to collect

\section{Anaesthetic considerations}

Suitable under local anaesthesia or sedation

Short operative time

Minimal cervical dilatation

Postoperative pain easily controlled with oral analgesia

\section{Economic consideration}

Can be used in outpatient or day-stay unit

Low capital cost

Low cost per treatment

Durable and easily sterilisable equipment permitting several treatments to be done in a single clinic session

Financial stable company to provide long-term product support 


\section{Comparison of FEAT'S and SEAT'S}

A systematic review Garside et al., 2005) compared MEA and TBEA with TCRE and RB. ${ }^{17}$ No significant differences were found between the FEAT's and SEATs in terms of amenorrheoa,bleeding patterns, premenstrual symptoms,patient satisfaction and quality of life.However,MEA and TBEA had significant shorter operating times. Currently there are no head to head trials of MEA and TBEA.There is not enough evidence in the differences in the clinical effectiveness of these two techniques.

\section{Need for further surgery}

In 1999 a randomized trial by the Aberdeen Endometrial Ablation Trial Group reported outcome at four years in patients managed by endometrial ablation who would otherwise have had a hysterectomy. Outcome at four years showed that two out of every five women allocated to endometrial ablation received further surgery, either hysterectomy or reablation. Hysterectomy was still avoided in $76 \%$ of women. ${ }^{18}$ In 2005 a randomized comparison of MEA and TCRE revealed a 16\% hysterectomy rate for MEA and 25\% for TCRE at five years. ${ }^{19}$

Table III-V lists the indications and contra-indicaitons of EA as well as the item list for preoperative assessment.

\section{Table III: Indications for endometrial ablation}

Disabling intrauterine bleeding for disruption of lifestyle, convenience, or unexplained bleeding on hormone replacement therapy (ACOG

Technical Bulletin, February 1990)

Failed traditional therapies

Contraindications to medical treatment

Poor surgical risks for hysterectomy

To preserve the uterus

\section{Table IV: Contraindications}

Relative:
Endometrial hyperplasia
Dysmenorrhoea
Chronic pelvic pain
Premenstrual syndrome
Multiple or large uterine fibroids
Enlarged uterus(more than $12 \mathrm{~cm}$ cavity length)
Uterine prolapse
Genital tract malignancy
Women wishing to preserve fertility
Intrauterine pregnancy
Women expecting amenorrhoea as an outcome
Acute pelvic inflammatory disease

\section{Absolute}

\section{Table V: Preablation patient assessment}

Patient counselling - she should have completed her family and be prepared for results that fall short of amenorrheoa (i.e. suitable for women who wish to maintain fertility)

Complete history

Detailed physical and pelvic examination

Papanicolaou smear

Pelvic imaging (sonography, MRI, CT scan)

Prior endometrial sample to rule out abnormal endometrial histology (office biopsy, D \& C, saline infusion sonohysterography)

Prior hysteroscopy to ensure the absence of major intrauterine pathology i.e. all organic and structural causes of UAB should be excluded

\section{Endometrial priming}

It is easier and faster to destroy or resect a thin endometrium. A thin endometrium is associated with fewer intraoperative and postoperative complications, reduces fluid absorption and may result in fewer failure rates. Endometrial thinning agents include $\mathrm{GnRh}$ analogues, progestins, danazol and oral contraceptives

Surgery is more effective and efficient when performed in the immediate postmenstrual phase, following curretage of the endometrium or pharmacologic thinning agents. ${ }^{20}$ It is interesting that NICE does not recommend endometrial thinning agents before TBEA. ${ }^{21}$

\section{Complications associated with global EA}

The US Food and Drug Administration's Manufacturer and User Facility Device Experience (MAUDE) database explored complications associated with SEATs. They noted a discrepancy in reported complications between published medical literature and the database reports. To date only few and minor complications have been reported in the medical literature. These include haemorrhage, pelvic inflammatory disease, endometritis, first degree skin burns, hematometra, vaginitis and cystits. A Medline search revealed 27 studies of which 18 reported no complications. Of the nine studies reporting complications, there were two cases of haemorrhage, one case of pelvic inflammatory disease, twenty cases of endometritis, two cases of first degree burns, nine cases of hematometra and sisteen cases of vaginitis and cystitis. Specifically no cases of uterine perforation or thermal bowel injury were reported. The Cochrane database revealed no additional cases.

The MAUDE database revealed thirty cases of uterine perforations, eight cases of thermal bowel injury and one case of necrotising fasciitis. ${ }^{22}$ (Numerator - worldwide complications and denominator - US sales). Thus physicians are encouraged to review the MAUDE database when considering the use of new medical devices.

\section{Pregnancy following EA}

The reported rate for pregnancy post EA is $0,65 \%{ }^{23}$ It is generally assumed that pregnancy is unlikely following EA. Potential complications of pregnancy following EA must be anticipated. The patient must be counseled regarding the increased risk of miscarriage, antepartum haemorrhage, intrauterine growth restriction, preterm delivery, perinatal mortality and abnormal placentation necessitating hysterectomy. It is vital that an informed decision is made.

Other issues relating to EA include treating patients with fibroid uteri. MEA was allowed to include patients with submucosal or intracavitary fibroids up to $3 \mathrm{~cm}$ in diameter. There are different views concerning this approach. All five devices that are FDA approved have been granted the treatment of submucosal or intracavitary fibroids as an inclusion criteria for therapy. The efficacy of this approach still needs to be established. EA is not a sterilization procedure. Patients desiring future fertility should not undergo the procedure. A Post-EA syndrome has been described in patients with prior sterilization and in those who have a laparoscopic sterilization at the time of EA.

\section{Evidence for endometrial ablation}

Patient preference- A recent study at a teaching hospital in the Netherlands ${ }^{24}$ explored patient preferences for endometrial ablation, a levonorgetrel-releasing intrauterine device, or 
hysterectomy. This study demonstrated that using a 50\% success rate of noninvasive techniques, the majority of women in the ablation group and in the levonorgestrel-releasing IUD group would choose a less invasive technique than hysterectomy. Avoidance of major surgery, combined with short or no hospitalization and quick recovery are major advantages of noninvasive treatment.

Cost analysis: The Markov model assessed the cost effectiveness of six hypothetical cohorts of women with heavy menstrual bleeding treated separately by TBEA, MEA, TCRE, TCRE and RB, RB or hysterectomy. The model calculated the incremental cost utility between different options over 10 years and concluded that the SEATs (TBEA and MEA) are more cost effective than the FEATs. Hysterectomy was more expensive but accrued more quality adjusted life years (QALYs) than all other EA methods. A QALY is a year of life adjusted for its value (quality) where a value of one is full health, and a value of 0 is death.

Other advantages include no surgical incision or organ removal. It can also be performed in an outpatient setting. The patient's hormonal status is unaffected. It also allows patients to return to normal activities with a day or two postoperatively versus four to six weeks posthysterectomy.

Patient satisfaction: In the Aberdeen Endometrial Ablations randomized Trials patient satisfaction was $89 \%$ in the hysterectomy group and $80 \%$ in the ablation group. The Cochrane database of systematic reviews also show greater patient satisfaction in the hysterectomy group.

Morbidity: The risk of serious complications from abdominal and vaginal hysterectomy has been reported in a number of studies. In a multicenter, prospective, nonrandomized study that included 1851 patients the perioperative death rate was 0,1\% for abdominal hysterectomy and $0,2 \%$ for vaginal hysterectomy. ${ }^{25}$ For women between 35-44 years who have a hysterectomy for benign conditions, the risk of dying is 3 per 10,000 women, and for women of all ages the risk of dying is 6 per 10,000.

The Cochrane database found EA to be effective and cheaper alternative to hysterectomy although retreatment is sometimes needed.

\section{Conclusion}

Choosing the best treatment for the patient is complex and ideally should involve balancing patient wishes, expected outcomes, complications, cost effectiveness and quality of life issues. The reversibility, contraceptive benefit, easier access, lower cost and opportunity for treatment in clinic setting with the LNG-IUS is an attractive option. If surgery is contemplated EA offers an effective conservative alternative to the more radical surgery through hysterectomy.

\section{References}

1. David C 1908: Lendoscopie uterine (hysteroscopy) applications au diagnostic et au traitment des affections intrauterines. In: Jacques $G$ (ed) These de Paris. Paris, 132

2. Vilos $G A$, Lefebvre G, Graves G. Guidelines for the management of abnormal uterine bleeding. SOGC Clinical Practice guideline No 106 Aug 2002. J Obstet Gynecol Can 2001;23:704-709.

3. Hallberg L, Hogdahl A, Nilsson L, Rybo G. Menstrual blood loss: a population study. Variations at different ages and attempts to define normality. Acta Obstet Gynaecol Scand 1996;45:320-351

4. Vessey M, Villard-Mackintosh L, McPherson K, Coulter A, Yeates D. The epidemiology of hysterectomy: findings in a large cohort study. Br J
Obstet Gynaecol 1992;99:402-407

5. Diane D. League. Endometrial Ablation as an alternative to hysterectomy. AORN J 2003;77:321-345.

6. Cooper JM, Erickson ML. Global endometrial ablation technologies. Obstet Gynecol Clin N Am 2005;27:385-396.

7. Chen BH, Giudice LC. Dysfunctional uterine bleeding. Western J Med 1998:169;280

8. Anderson JK, Rybo G. Levonorgestrel-releasing intrauterine device in the treatment of menorrhagia. Br J Obstet Gynaecol 1990;97:690-694.

9. Hurskainen R, Teperi J, Rissanen P, et al. Quality of life and cost effectiveness of levonorgestrel-releasing intrauterine system versus hysterectomy for treatment of menorrhagia: a randomised trial. Lancet 2001;357:273-277.

10. Barrington JW, Arunkalaivanan AS, Abdel Fattah M. Comparison between the levonorgestrel intrauterine system (LNG-IUS) and thermal balloon ablation in the treatment of menorrhagia. Eur J Obstet Gynecol Reprod Biol 2003; 108:72-74.

11. Lethaby A, Cooke I, Rees M. Progesterone/progestogen releasing intrauterine systems versus placebo or any other medication for heavy menstrual bleeding. Cochrane Database system Rev 2000:CD002126.

12. Garry R. Evidence and techniques in endometrial ablation: consensus. Gynaecol Endosc 2002;1 1:5-17.

13. A national survey of complications of endometrial destruction for menstrual disorders: the MISTLETOE study. Br J Obstet Gynaecol 1997;104:1351-1359.

14. Thermal Balloon and Rollerball Ablation to treat menorrhagia: A multicenter comparison. Obstet Gynecol 1998;92:98-103.

15. Nazar $N$ et al. Uterine endometrial thermal balloon therapy for the treatment of menorrhagia: longterm multicentre follow-up study. Hum Rep 2003; 18(5):1082-1087.

16. Thomas J. Clark,Janesh K. Gupta. Outpatient thermal balloon ablation of the endometrium. Fertil Steril 2004;82(5):1395-1401.

17. Garside Ruth, Stein Ken, Wyatt Katrina, Round Ali. Microwave and thermal balloon ablation for heavy menstrual bleeding: a systematic review. Br J Obstet Gynaecol 2005; 105:12-23.

18. Aberdeen Endometrial Ablation Trials Group. A randomized trial of endometrial ablation versus hysterectomy for the treatment of dysfunctional uterine bleeding: outcome at four years. Br J Obstet Gynaecol 1999;106:360-366.

19. Cooper Kevin G, Baine Christine, Lawrie Lisa, Parkin David E. A randomised comparison of microwave endometrial ablation with trancervical resection of the endometrium; follow up at a minimum of five years. Br J Obstet Gynaecol 2005; 1 12:470-475.

20. Brooks PG, Sarden SP, Davos J. Hormonal inhibition of the endometrium for resectoscopic endometrial ablation. Am J Obstet Gynecol 1991;164:1601-1608

21. NICE Technology Appraisal 78. Fluid-filled thermal balloon and microwave endometrial ablation techniques for heavy menstrual bleeding.

22. Shawn E, Gutcheff, Howard T. Sharp. Complications associated with Global Endometrial Ablation: The utility of the MAUDE Database. Obstet Gynecol 2003;102:1278-1282.

23. Hare AA, Olah KS. Pregnancy following endometrial ablation: a review article. J Obstet Gynaecol 2005;25(2):108-1 14.

24. Bourdrez Petra, Bonger Marlies Y, Ben WJ Mol. Treatment of dysfunctional uterine bleeding: patient preferences for endometrial ablation, a levonorgestrel releasing intrauterine device or hysterectomy. Fertil Steril 2004;82(1):160-166.

25. Dicker R, Greenspan J, Strauss L et al. Complications of abdominal and vaginal hysterectomy among women of reproductive age in the United States. Am J Obstet Gynecol 1982;144:841-848. 